\title{
ON STRONGLY SUMMABLE ULTRAFILTERS AND UNION ULTRAFILTERS
}

\author{
ANDREAS BLASS AND NEIL HINDMAN
}

\begin{abstract}
We prove that union ultrafilters are essentially the same as strongly summable ultrafilters but ordered-union ultrafilters are not. We also prove that the existence of ultrafilters of these sorts implies the existence of $P$-points and therefore cannot be established in ZFC.
\end{abstract}

1. Introduction. The purpose of this paper is to clarify the connections between the strongly summable ultrafilters introduced in [9] and the union ultrafilters and ordered-union ultrafilters introduced in [3]. We show that strongly summable ultrafilters and union ultrafilters are essentially the same in that every ultrafilter of either sort is isomorphic to one of the other sort via an isomorphism that (almost everywhere) respects the operations of summation and union. We also show that the existence of such ultrafilters, deduced in $[9,3]$ from the continuum hypothesis $(\mathrm{CH})$ or Martin's axiom (MA), cannot be deduced in ZFC alone, for it implies the existence of $P$-point ultrafilters. Finally, we show that union ultrafilters and ordered-union ultrafilters are not essentially equivalent; we construct, using $\mathrm{CH}$ or MA, a union ultrafilter that is not isomorphic to any ordered-union ultrafilter via an isomorphism that respects unions.

For any set $A$ of natural numbers, let $\mathrm{FS}(A)$ be the set of all sums of nonempty finite subsets of $A$. An ultrafilter on the set $\omega$ of natural numbers is strongly summable if it has a base consisting of sets of the form $\mathrm{FS}(A)$, with $A$ infinite.

We write $\mathbf{F}$ for the set of all finite nonempty subsets of $\omega$. For $A \subseteq \mathbf{F}$, let $\mathrm{FU}(A)$ be the set of all unions of nonempty finite subsets of $A$. An ultrafilter on $\mathbf{F}$ is a union ultrafilter if it has a base consisting of sets of the form $\mathrm{FU}(A)$, with $A$ an infinite family of pairwise disjoint elements of $\mathbf{F}$. It is an ordered-union ultrafilter if it has a base consisting of sets $\mathrm{FU}(A)$ where $A=\left\{a_{0}, a_{1}, \ldots\right\} \subseteq \mathbf{F}$ and, for each $i$, $\max \left(a_{i}\right)<\min \left(a_{i+1}\right)$, which we also write as $a_{i}<a_{i+1}$.

Eric van Douwen observed that the finite sum theorem [7, Theorem 3.1] and the construction in [6] establish the existence of strongly summable ultrafilters under $\mathrm{CH}$. He asked whether this existence could be established in ZFC alone. Our Theorem 3 below answers this question negatively.

Motivated by van Douwen's question, the second author analyzed strong summability and related concepts (including idempotence) in [9]. Motivated by this analysis

Received by the editors March 14, 1986.

1980 Mathematics Subject Classification. Primary 03E05; Secondary 03E35, 03E50 
and by Baumgartner's proof [1] of the finite unions theorem [7, Corollary 3.3], the first author introduced both union and ordered-union ultrafilters in [3] but studied the ordered-union ones almost exclusively. It was pointed out, in [3, §5], that the function $\mathbf{F} \rightarrow \omega$ sending each $s \in \mathbf{F}$ to $\sum_{i \in s} 2^{i}$ sends union ultrafilters to strongly summable ones, but questions about the converse and about the relationship between union and ordered-union ultrafilters were left open. Theorems 1 and 4 below provide answers to several of these questions.

In view of the crucial role played by the operation of addition on $\omega$ (resp. union on F) in the definition of strongly summable (resp. union and ordered-union) ultrafilters, it seems reasonable to adopt a strengthened notion of isomorphism for such ultrafilters, requiring the isomorphism to respect these operations. We say that an ultrafilter $\mathscr{U}$ on $\omega$ and an ultrafilter $\mathscr{V}$ on $\mathbf{F}$ are additively isomorphic if there exist $A \subseteq \omega$ and pairwise disjoint $B \subseteq \mathbf{F}$ such that $\mathrm{FS}(A) \in \mathscr{U}, \mathrm{FU}(B) \in \mathscr{V}$, and there exists a bijection $f: \mathrm{FS}(A) \rightarrow \mathrm{FU}(B)$ such that

(i) $f$ maps $A$ onto $B$,

(ii) for every finite nonempty $F \subseteq A, f\left(\sum_{a \in F} a\right)=\bigcup_{a \in F} f(a)$, and

(iii) $f(\mathscr{U})=\mathscr{V}$.

We remark, in connection with (iii), that the usual definition of $f(\mathscr{U})$, namely

$$
f(\mathscr{U})=\left\{X \subseteq \mathbf{F} \mid f^{-1}(X) \in \mathscr{U}\right\},
$$

makes sense even though $f$ is defined only on $\operatorname{FS}(A)$, not on all of $\omega$; if $f$ were extended arbitrarily to have domain $\omega, f(\mathscr{U})$ would be unchanged.

Additive isomorphism is defined analogously between two ultrafilters on $\omega$ (replace all unions by sums in the preceding definition), between two ultrafilters on $\mathbf{F}$ (replace all sums by unions), and from an ultrafilter on $\mathbf{F}$ to one on $\omega$ (interchange sums and unions, or just replace $f$ by its inverse).

\section{Strongly summable and union ultrafilters are equivalent.}

THEOREM 1. Every strongly summable ultrafilter is additively isomorphic to a union ultrafilter. Every union ultrafilter is additively isomorphic to a strongly summable ultrafilter.

Proof. The second assertion is easy because the function $\mathbf{F} \rightarrow \omega-\{0\}: s \mapsto$ $\sum_{a \in s} 2^{a}$ satisfies the additivity requirements with $A=\{\{n\} \mid n \in \omega\}$ ( $\operatorname{so} \operatorname{FU}(A)=\mathbf{F}$ ) and $B=\left\{2^{n} \mid n \in \omega\right\}$ (so $\operatorname{FS}(B)=\omega-\{0\}$ ). It sends sets of the form $\operatorname{FU}(S)$ to sets of the form $\operatorname{FS}(T)$ and therefore sends every union ultrafilter to a strongly summable one.

To prove the converse, let a strongly summable $\mathscr{U}$ be given.

Lemma 1A. There is a set $X=\left\{x_{0}, x_{1}, \ldots\right\}$ such that $\mathrm{FS}(X) \in \mathscr{U}$ and, for each $n$, $x_{n}>2 \sum_{k<n} x_{k}$.

Proof. Partition $\omega-\{0\}$ into two pieces, $C_{0}$ and $C_{1}$, by putting $x$ into $C_{0}$ if and only if $2^{n} \leqslant x<2^{n+1}$ for some even $n$, i.e., the number of digits in the binary notation for $x$ is odd. One of the two pieces is in $\mathscr{U}$, because $\mathscr{U}$ is an ultrafilter, and strong summability implies that this piece includes some $\operatorname{FS}(X) \in \mathscr{U}$. $X$ cannot 
contain two elements with the same number of binary digits, for then their sum would be one digit longer and could not belong to the same partition class. So $X=\left\{x_{0}, x_{1}, \ldots\right\}$ where each $x_{n}$ has at least two more binary digits than any earlier $x_{k}$. It follows that $x_{n}$ has at least one more binary digit than $\sum_{k<n} x_{k}$; but then it has at least two more, since both $x_{n}$ and $\sum_{k<n} x_{k}$ are in $\operatorname{FS}(X)$ and therefore in the same $C_{i}$. Thus, $x_{n}>2 \sum_{k<n} x_{k}$.

Lemma 1B. Let $X$ be as in the preceding lemma. No two distinct finite subsets of $X$ have the same sum.

Proof. We use only that $x_{n}>\sum_{k<n} x_{k}$, without the factor 2. Suppose the lemma were false, and let finite subsets $F, G$ of $X$ be a counterexample for which the common sum is as small as possible. Let $x_{n}$ be the largest element of $F \cup G$. If it is in both $F$ and $G$, then $F-\left\{x_{n}\right\}$ and $G-\left\{x_{n}\right\}$ constitute a counterexample with smaller sum, contradicting minimality. If it is in only one of $F$ and $G$, say $F$, then all elements of $G$ are $x_{k}$ with $k<n$, so

$$
\sum F \geqslant x_{n}>\sum_{k<n} x_{k} \geqslant \sum G
$$

so $F$ and $G$ do not constitute a counterexample.

A very similar argument gives the following lemma, in which the factor 2 in Lemma $1 \mathrm{~A}$ is used.

Lemma 1C. Let $X$ be as in Lemma 1A. No number can be expressed in two distinct ways as a linear combination of elements of $X$ with coefficients equal to 1 and 2 .

Proof. Suppose the lemma were false, and let $z$ be the smallest counterexample. Fix two distinct expressions for $z$ of the required form. As in the preceding proof, let $x_{n}$ be the largest element of $X$ that occurs (with either coefficient) in either of these expressions, and obtain a contradiction to the minimality of $z$ if $x_{n}$ occurs in both of the expressions. Then $z \geqslant x_{n}$ because $x_{n}$ occurs in one of the expressions. But the other expression consists of $x_{k}$ 's with $k<n$, with coefficients at most 2, so the number $z$ that it represents is $\leqslant 2 \sum_{k<n} x_{k}<x_{n}$, a contradiction.

Lemma 1B allows us to define a function $f: \operatorname{FS}(X) \rightarrow \mathrm{FU}(Y)$, where $Y=$ $\{\{x\} \mid x \in X\}$, by $f\left(\sum_{x \in F} x\right)=F$ for all nonempty finite $F \subseteq X$. This $f$ clearly satisfies the first two requirements for an additive isomorphism, so Theorem 1 will be proved if we show that $f(\mathscr{U})$ is a union ultrafilter. Since $\mathscr{U}$ has a basis of sets $\mathrm{FS}(A) \subseteq \mathrm{FS}(X), f(\mathscr{U})$ has a basis of corresponding sets $f[\mathrm{FS}(A)]$.

Lemma 1D. Let $X$ and $f$ be as above. If $\mathrm{FS}(A) \subseteq \mathrm{FS}(X)$, then $f[A]$ consists of pairwise disjoint sets.

Proof. Suppose we had distinct $a, b \in A$ such that $f(a)=F$ and $f(b)=G$ were not disjoint. By adding the representations $a=\sum_{x \in F} x$ and $b=\sum_{x \in G} x$ (which come from the definition of $f$ ), we would obtain a representation of $a+b$ as a linear combination of elements of $F \cup G \subseteq X$, with coefficients 1 and 2 (for 
elements $x$ of the symmetric difference $F \Delta G$ and the intersection $F \cap G$, respectively). As $F$ and $G$ are not disjoint, at least one coefficient is 2. But $a+b \in \mathrm{FS}(A)$ $\subseteq \mathrm{FS}(X)$, so $a+b$ also has a representation as a finite sum of elements of $X$ without 2 as a coefficient. This contradicts Lemma 1C.

Now consider the action of $f$ on a typical element $b$ of $\mathrm{FS}(A)$. Such a $b$ is the sum of a finite subset $F$ of $A$, and each $a \in F$ is in turn the sum of the finite subset $f(a)$ of $X$. By the last lemma, these sets $f(a)$ are pairwise disjoint, so $b$ is the sum of their union. That is, $f(b)=\bigcup_{a \in F} f(a)$. Therefore, $f[\mathrm{FS}(A)]=\mathrm{FU}(f[A])$.

We have shown that $f(\mathscr{U})$ has a basis of sets of the form $\mathrm{FU}(f[A])$ with $f[A]$ a pairwise disjoint subfamily of $\mathbf{F}$. So $f(\mathscr{U})$ is a union ultrafilter.

3. Union ultrafilters yield $P$-points. Recall that a nonprincipal ultrafilter on $\omega$ is a $P$-point if every function on $\omega$ becomes finite-to-one or constant when restricted to some set in the ultrafilter. Observe that one could replace "constant" here with "bounded", for any ultrafilter that contains $\{n \mid f(n) \leqslant k\}$ must also contain $\{n \mid f(n)=j\}$ for one of the finitely many $j \leqslant k$.

We write $\max$ (resp. min) for the function $\mathbf{F} \rightarrow \omega$ that sends each set $s \in \mathbf{F}$ to its largest (resp. smallest) member. Thus, if $\mathscr{U}$ is an ultrafilter on $\mathbf{F}$ then $\max (\mathscr{U})$ and $\min (\mathscr{U})$ are ultrafilters on $\omega$.

THEOREM 2. If $\mathscr{U}$ is a union ultrafilter, then $\max (\mathscr{U})$ and $\min (\mathscr{U})$ are P-points.

Proof. We first treat $\max (\mathscr{U})$. Let $f: \omega \rightarrow \omega$ be given. Because $\mathscr{U}$ is a union ultrafilter, there is an infinite pairwise disjoint family $A \subseteq \mathbf{F}$ such that $\mathrm{FU}(A) \in \mathscr{U}$ and either all or none of the sets $s \in \mathrm{FU}(A)$ satisfy $f(\max (s)) \leqslant \min (s)$.

Case 1. For all $s \in \mathrm{FU}(A), f(\max (s)) \leqslant \min (s)$.

Let $a$ be the element of $A$ with the smallest $\min (a)$. For all $b \in A$ with $\max (b) \geqslant \max (a)$, we have, since $a \cup b \in \mathrm{FU}(A)$,

$$
f(\max (b))=f(\max (a \cup b)) \leqslant \min (a \cup b)=\min (a) .
$$

Thus, $f$ is bounded by $\min (a)$ on a cofinite subset of

$$
\max [A]=\max [\mathrm{FU}(A)] \in \max (\mathscr{U}) .
$$

Case 2. For all $s \in \mathrm{FU}(A), f(\max (s))>\min (s)$.

Let any $k \in \omega$ be given. If $b \in A$ and $f(\max (b))=k$, then $\min (b)<k$. Thus $f$ takes the value $k$ only finitely often on $\max [A]=\max [\mathrm{FU}(A)] \in \max (\mathscr{U})$. As $k$ was arbitrary, $f$ is finite-to-one on a set in $\max (\mathscr{U})$.

This completes the proof that $\max (\mathscr{U})$ is a $P$-point.

We turn to $\min (\mathscr{U})$. Again, let $f: \omega \rightarrow \omega$ be given. For each $s \in \mathbf{F}$, let $h(s)$ be the number of consecutive pairs $(x, y)$ from $s$ (i.e., $x<y$, both are in $s$, and no number between $x$ and $y$ is in $s$ ) such that $f(y)<x$. Because $\mathscr{U}$ is a union ultrafilter, there is an infinite pairwise disjoint family $A \subseteq \mathbf{F}$ such that $\mathrm{FU}(A) \in \mathscr{U}$ and $h(s)$ has the same parity (i.e., the same residue modulo 2 ) for all $s \in \mathrm{FU}(A)$.

Suppose $a, b \in A$ and $\max (a)<\min (b)$. Then the pairs counted by $h(a \cup b)$ are those counted by $h(a)$, those counted by $h(b)$, and perhaps the pair $(\max (a), \min (b))$; the latter pair is included if and only if $f(\min (b))<\max (a)$. 
Therefore,

$$
h(a \cup b)=h(a)+h(b)+ \begin{cases}1 & \text { if } f(\min (b))<\max (a), \\ 0 & \text { if } f(\min (b)) \geqslant \max (a) .\end{cases}
$$

Case 1. $h(s)$ is odd for all $s \in \mathrm{FU}(A)$.

Then the last term in $(*)$ must be 1 , not 0 , and so $f(\min (b))<\max (a)$ for all $a, b$ as above. If we fix $a$ and let $b$ vary, we find that $f$ is bounded by $\max (a)$ on $\{\min (b) \mid b \in A$ and $\max (a)<\min (b)\}$, a cofinite subset of $\min [A]=\min [\mathrm{FU}(A)]$ $\in \min (\mathscr{U})$.

Case 2. $h(s)$ is even for all $s \in \mathrm{FU}(A)$.

Now the last term in $(*)$ must be 0 , and so $f(\min (b)) \geqslant \max (a)$ for all $a, b$ as above. Let an arbitrary $k \in \omega$ be given. Fix an $a \in A$ with $\max (a)>k$. Then the only $\min (b) \in \min [A]$ for which we can have $f(\min (b))=k$ are those such that $a$, $b$ are not as above, i.e., those with $\min (b) \leqslant \max (a)$. As $a$ is fixed, there are only finitely many $\operatorname{such} \min (b)$. So $f$ is finite-to-one on $\min [A]$.

This completes the proof that $\min (\mathscr{U})$ is also a $P$-point.

COROLlary. If $\mathscr{U}$ is an ordered-union ultrafilter, then $\min (\mathscr{U})$ and $\max (\mathscr{U})$ are selective ultrafilters.

Proof. Combine the theorem with Proposition 3.9 of [3].

THEOREM 3. It is consistent relative to ZFC that there are no union ultrafilters and no strongly summable ultrafilters.

Proof. For union ultrafilters, combine Theorem 2 with Shelah's theorem [11(VI §4), 12] that it is consistent relative to ZFC that there are no $P$-points. For strongly summable ultrafilters, invoke also Theorem 1.

When the typing of this paper was nearly completed, we learned from Pierre Matet of his unpublished paper, Some filters of partitions, in which he showed that, for union ultrafilters $\mathscr{U}, \max (\mathscr{U})$ is a rapid $P$-point. The same paper also anticipates some of the results in [3].

4. Union and ordered-union differ. In this section we show, under suitable set-theoretic hypotheses, that the class of union ultrafilters is essentially larger than that of ordered-union ultrafilters. We first give a proof assuming the continuum hypothesis. Afterward, we indicate how to modify this proof to get the same results assuming only Martin's axiom or the even weaker hypothesis $P(c)$.

THEOREM 4. Assume CH. There is a union ultrafilter that is not additively isomorphic to any ordered-union ultrafilter.

Recall that an ultrafilter on $\omega$ is called a $Q$-point if every finite-to-one function on $\omega$ becomes one-to-one when restricted to a suitable set in the ultrafilter. It was shown in [3, Proposition 3.9] that if $\mathscr{U}$ is an ordered-union ultrafilter then both $\min (\mathscr{U})$ and $\max (\mathscr{U})$ are $Q$-points. Thus, to prove Theorem 4 , it more than suffices to prove the following. 
TheOREM 4'. Assume CH. There is a union ultrafilter such that no additively isomorphic ultrafilter is sent to a Q-point by either $\min$ or $\max$.

We can simplify our task further by showing that only a special sort of additive isomorphism must be considered. If $g: \omega \rightarrow \omega$ is one-to-one, then the function $\dot{g}$ : $\mathbf{F} \rightarrow \mathbf{F}$ sending each $s \in \mathbf{F}$ to its image under $g$ (i.e., $\dot{g}(s)=g[s]$ ) preserves all unions and therefore sends every ultrafilter on $\mathbf{F}$ to an additively isomorphic one. The following lemma allows us to restrict our attention to these isomorphisms $\dot{g}$.

Lemma 4A. If union ultrafilters $\mathscr{U}$ and $\mathscr{V}$ are additively isomorphic, then there are one-to-one functions $g$ and $h$ from $\omega$ to $\omega$ such that $\min (\dot{g}(\mathscr{U}))=h(\min (\mathscr{V}))$ and $\max (\dot{g}(\mathscr{U}))=h(\max (\mathscr{V}))$.

Proof. Observe first that every union ultrafilter must contain the set $\{s \in \mathbf{F} \mid$ $\min (s) \neq \max (s)\}$, for the complementary set, the set of singletons, does not include $\mathrm{FU}(A)$ for any infinite $A$.

Let $A, B$, and $f$ be as in the definition of additive isomorphism from $\mathscr{U}$ to $\mathscr{V}$. By the preceding observation, we may replace $A$ and $B$ by subsets of $\mathrm{FU}(A)$ and $\mathrm{FU}(B)$ such that all the requirements on $A, B$, and $f$ remain satisfied and, furthermore, $\min (s) \neq \max (s)$ for all $s \in A \cup B$. Let $h: \omega \rightarrow \omega$ be any strictly order-preserving function with the additional property that, for each $s \in A$,

$$
h(\max (f(s)))-h(\max (f(s))-1)=|s|,
$$

the cardinality of $s$. (It is trivial to construct such an $h$ by induction.) The set of $|s|-1$ numbers strictly between $h(\max (f(s))-1)$ and $h(\max (f(s)))$, which are not in the range of $h$, will be called the gap associated to $s$. Notice that the gaps associated to different elements $s$ of $A$ are disjoint.

Define $g: \omega \rightarrow \omega$ as follows. For each $s \in A$, set $g(\min (s))=h(\min (f(s)))$ and $g(\max (s))=h(\max (f(s)))$. Then let $g$ map the remaining $|s|-2$ members of $s$ to $|s|-2$ distinct members of the gap associated to $s$. One element of each gap is not used here, so $g$ can be extended to a one-to-one function on all of $\omega$.

Consider any $s \in \mathrm{FU}(A)$, say $s=s_{0} \cup \cdots \cup s_{k}$ with each $s_{i} \in A$. Then $g[s]$ consists of the elements $h\left(\min \left(f\left(s_{i}\right)\right)\right), h\left(\max \left(f\left(s_{i}\right)\right)\right)$, and some members of the gaps associated to the $s_{i}$. As $h$ is order-preserving, $\min (g[s])$ is $h\left(\min \left(f\left(s_{i}\right)\right)\right)$ for some $i$, namely the $i$ with the smallest $\min \left(f\left(s_{i}\right)\right)$. That is, since $f(s)=f\left(s_{0}\right)$ $\cup \cdots \cup f\left(s_{k}\right), \min (\dot{g}(s))=h(\min (f(s)))$. Similarly, $\max (\dot{g}(s))=h(\max (f(s)))$. As these equations hold for all $s \in \mathrm{FU}(A)$ and $\mathrm{FU}(A) \in \mathscr{U}$, it follows that

$$
\min (\dot{g}(\mathscr{U}))=h(\min (f(\mathscr{U})))=h(\min (\mathscr{V}))
$$

and similarly $\max (\dot{g}(\mathscr{U}))=h(\max (\mathscr{V}))$.

Since the $h$ in the lemma is one-to-one, $\min (\dot{g}(\mathscr{U}))=h(\min (\mathscr{V}))$ is a $Q$-point if and only if $\min (\mathscr{V})$ is a $Q$-point, and similarly with max in place of min. Therefore, to prove Theorem $4^{\prime}$, it suffices to prove the following.

THEOREm 4". Assume CH. There is a union ultrafilter $\mathscr{U}$ such that, for every one-to-one $g: \omega \rightarrow \omega$, neither $\max (\dot{g}(\mathscr{U}))$ nor $\min (\dot{g}(\mathscr{U}))$ is a Q-point. 
Proof. The ultrafilter $\mathscr{U}$ will be constructed by a transfinite recursion of length $\aleph_{1}$, in which each step will take care of one subset $X$ of $\mathbf{F}$ and one injective function $g: \omega \rightarrow \omega$. To take care of $X$ means to ensure that either $X$ or its complement $\mathbf{F}-X$ belongs to $\mathscr{U}$. To take care of $g$ means to select a finite-to-one $f$ such that $f$ will not be one-to-one on any set in $\max (\dot{g}(\mathscr{U}))$ or $\min (\dot{g}(\mathscr{U}))$. Thus, the choice of $f$ imposes a constraint on what sets we can put into $\mathscr{U}$ at later stages of the construction; in fact, it will be necessary to strengthen this constraint in order to make the induction work. At any stage of the construction we are subject to countably many such constraints, arising from the countably many carlier stages. We now formulate what these constraints, appropriately strengthened, look like.

Let $\mathscr{C}$ be any family of pairs $(f, g)$ of functions $\omega \rightarrow \omega$ such that each of the first components $f$ is finite-to-one and each of the second components $g$ is one-to-one. Let $\mathscr{H}$ be a finite subfamily of $\mathscr{C}$, and let $m \in \omega$. A subset $S$ of $\mathbf{F}$ will be called ( $\mathscr{H}, m$ )-adequate if there exist $m$ pairwise disjoint sets $s_{0}, \ldots, s_{m-1}$ such that

(i) $\mathrm{FU}\left(\left\{s_{0}, \ldots, s_{m-1}\right\}\right) \subseteq S$, and

(ii) for all $i, j<m$, and all $(f, g) \in \mathscr{H}$,

$$
f\left(\max \left(\dot{g}\left(s_{i}\right)\right)\right)=f\left(\max \left(\dot{g}\left(s_{j}\right)\right)\right) \text { and } f\left(\min \left(\dot{g}\left(s_{i}\right)\right)\right)=f\left(\min \left(\dot{g}\left(s_{j}\right)\right)\right) \text {. }
$$

$S$ is $\mathscr{C}$-adequate if it is $(\mathscr{H}, m)$-adequate for all finite $\mathscr{H} \subseteq \mathscr{C}$ and all $m \in \omega$. We intend to build $\mathscr{U}$ so that all sets in it are $\mathscr{C}$-adequate for a $\mathscr{C}$ such that every one-to-one $g: \omega \rightarrow \omega$ occurs as the second component of some pair in $C$; as will be shown later, this will ensure that neither $\min (\dot{g}(\mathscr{U})) \operatorname{nor} \max (\dot{g}(\mathscr{U}))$ is a $Q$-point. The next few lemmas will allow us to take care of a subset $X$ of $\mathbf{F}$ without losing the adequacy achieved at previous stages of our construction.

LEMmA 4B. For each $m \in \omega$ there exists $M \in \omega$ such that, if $\mathscr{H}$ is any finite family as above and if an $(\mathscr{H}, M)$-adequate set is partitioned into two pieces, then one of the pieces is $(\mathscr{H}, m)$-adequate.

Proof. By a partition theorem of Graham and Rothschild, [5, Corollary 3] for each $m \in \omega$ there exists $M \in \omega$ such that, if the family of nonempty subsets of $M=\{0,1, \ldots, M-1\}$ is partitioned into two pieces, then there are $m$ pairwise disjoint nonempty subsets $t_{0}, \ldots, t_{m-1}$ of $M$ all of whose nonempty unions lie in the same piece of the partition. Now suppose that an $(\mathscr{H}, M)$-adequate set $S$ is partitioned into two pieces $S_{0}$ and $S_{1}$. Let $s_{0}, \ldots, s_{M-1}$ be as in the definition of $(\mathscr{H}, M)$-adequate, and partition the family of nonempty subsets of $M$ into $P_{0}$ and $P_{1}$ by putting $t \in P_{l}$ if and only if $\bigcup_{j \in t} s_{j} \in S_{l}$. By our choice of $M$, there are nonempty pairwise disjoint subsets $t_{0}, \ldots, t_{m-1}$ of $M$ all of whose nonempty unions lie in the same $P_{l}$. Then the sets

$$
s_{i}^{\prime}=\bigcup_{j \in t_{i}} s_{j}
$$

and all their nonempty unions lie in $S_{l}$. Furthermore, for each $i<m$ and each pair $(f, g) \in \mathscr{H}, \min \left(\dot{g}\left(s_{i}^{\prime}\right)\right)=\min \left(\dot{g}\left(s_{j}\right)\right)$ for a certain $j \in t_{i}$ (that can depend on $g$ ). Since $f\left(\min \left(\dot{g}\left(s_{j}\right)\right)\right)$ is independent of $j$ by clause (ii) in the definition of adequacy according to which the $s_{j}$ were chosen, it follows that $f\left(\min \left(\dot{g}\left(s_{i}^{\prime}\right)\right)\right)$ is independent 
of $i$. Of course the same argument works with max in place of min. Therefore, $s_{0}^{\prime}, \ldots, s_{m-1}^{\prime}$ witness the $(\mathscr{H}, m)$-adequacy of $S_{l}$.

LEMMA 4C. If a $\mathscr{C}$-adequate set is partitioned into two pieces, then at least one of the pieces is also $\mathscr{C}$-adequate.

Proof. Suppose not. Then one piece fails to be $\left(\mathscr{H}_{0}, m_{0}\right)$-adequate and the other fails to be $\left(\mathscr{H}_{1}, m_{1}\right)$-adequate, for certain finite $\mathscr{H}_{i} \subseteq \mathscr{C}$ and certain $m_{i} \in \omega$. Neither piece is $(\mathscr{H}, m)$-adequate, where $\mathscr{H}=\mathscr{H}_{0} \cup \mathscr{H}_{1}$ and $m=\max \left\{m_{0}, m_{1}\right\}$. By the preceding lemma, there is an $M$ such that the original set is not $(\mathscr{H}, M)$-adequate. But this contradicts the assumption that the original set is $\mathscr{C}$-adequate.

If we were merely trying to build an ultrafilter consisting of adequate sets, the preceding lemma would suffice. Since we need a union ultrafilter, we must work harder. We need adequate homogeneous sets of the form $\mathrm{FU}(A)$, and we get them by a variant of the proof $[4,8]$ of the finite sum theorem given by Glazer (building on an idea of Galvin).

Until further notice, assume that

$\mathscr{C}$ is a countable family of pairs $(f, g)$ of functions $\omega \rightarrow \omega$ with $f$ finite-to-one and $g$ one-to-one,

$A$ is a pairwise disjoint subfamily of $\mathbf{F}$, and

$\mathrm{FU}(A)$ is $\mathscr{C}$-adequate.

Let $\Phi$ be the collection of all filters $\mathscr{F}$ on $\mathbf{F}$ such that every set in $\mathscr{F}$ is $\mathscr{C}$-adequate and, for every finite $Q \subseteq A, \mathrm{FU}(A-Q) \in \mathscr{F}$. Let $\Psi$ be the collection of all ultrafilters in $\Phi$.

LEMMA 4D. $\Psi$ is nonempty.

Proof. From the $\mathscr{C}$-adequacy of $\mathrm{FU}(A)$, it follows that, for every finite $Q \subseteq A$, $\mathrm{FU}(A-Q)$ is also $\mathscr{C}$-adequate; indeed, if $Q$ has cardinality $q$, then among any $m+q$ pairwise disjoint elements of $\mathrm{FU}(A)$ witnessing $(\mathscr{H}, m+q)$-adequacy, at most $q$ will include members of $Q$, and the remaining $m$ will witness the $(\mathscr{H}, m)$ adequacy of $\mathrm{FU}(A-Q)$. Thus, $\Phi$ contains the filter generated by the sets $\mathrm{FU}(A-Q)$ for all finite $Q \subseteq A$. In particular, $\Phi$ is nonempty. Since $\Phi$ is clearly closed under unions of nonempty chains, Zorn's Lemma provides a maximal $\mathscr{F} \in \Phi$. We shall show that $\mathscr{F}$ is an ultrafilter and therefore in $\Psi$.

Let $X \subseteq \mathbf{F}$. We must show that either $X$ or $\mathbf{F}-X$ belongs to $\mathscr{F}$, so suppose that neither does. By the maximality of $\mathscr{F}$, the filters generated by $\mathscr{F} \cup\{X\}$ and by $\mathscr{F} \cup\{\mathbf{F}-X\}$ are not in $\Phi$, so each contains a set that is not $\mathscr{C}$-adequate. Shrinking these sets (which preserves inadequacy) if necessary, we may assume that they are $A_{0} \cap X$ and $A_{1}-X$ with $A_{0}, A_{1} \in \mathscr{F}$. Then $A_{0} \cap A_{1}$ can be partitioned into two sets $A_{0} \cap A_{1} \cap X \subseteq A_{0} \cap X$ and $A_{0} \cap A_{1}-X \subseteq A_{1}-X$, neither of which is $\mathscr{C}$ adequate. By Lemma $4 \mathrm{C}, A_{0} \cap A_{1}$ is not $\mathscr{C}$-adequate. But $A_{0} \cap A_{1} \in \mathscr{F}$, so this contradicts the fact that $\mathscr{F} \in \Phi$.

Let $\oplus: \beta \mathbf{F} \times \beta \mathbf{F} \rightarrow \beta \mathbf{F}$ be the canonical extension, to the Stone-Čech compactification $\beta \mathbf{F}$, of the operation of union $U: \mathbf{F} \times \mathbf{F} \rightarrow \mathbf{F}$. Depending on one's choice of conventions, the ultrafilter

$$
\mathscr{X}=\{X \subseteq \mathbf{F} \mid\{s \in \mathbf{F} \mid\{t \in \mathbf{F} \mid s \cup t \in X\} \in \mathscr{V}\} \in \mathscr{W}\}
$$


is either $\mathscr{V} \oplus \mathscr{W}$ or $\mathscr{W} \oplus \mathscr{V}$, and the operation $\oplus$ is continuous in one of its two arguments (but not in the other). With either convention, $\oplus$ is associative.

LEMMA 4E. $\Psi$ is closed under $\oplus$.

Proof. Assume that $\mathscr{V}, \mathscr{W} \in \Psi$ and let $\mathscr{X}$ be as displayed above; we verify that $\mathscr{X} \in \Phi$, for it is clearly an ultrafilter. First, suppose $X \in \mathscr{X}$. To show that $X$ is $\mathscr{C}$-adequate, we fix some finite $\mathscr{H} \subseteq \mathscr{C}$ and fix $m \in \omega$, and we show that $X$ is $(\mathscr{H}, m)$-adequate. Let

$$
X^{\prime}=\{s \in \mathbf{F} \mid\{t \in \mathbf{F} \mid s \cup t \in X\} \in \mathscr{V}\} .
$$

Then the assumption that $X \in \mathscr{X}$ means that $X^{\prime} \in \mathscr{W}$. As $\mathscr{W} \in \Psi, X^{\prime}$ must be $\mathscr{C}$-adequate and therefore $(\mathscr{H}, m)$-adequate. Fix $s_{0}, \ldots, s_{m-1}$ as in the definition of $(\mathscr{H}, m)$-adequacy of $X^{\prime}$. In particular, if $s \in \mathrm{FU}\left(\left\{s_{0}, \ldots, s_{m-1}\right\}\right)$ then $s \in X^{\prime}$, which means $\{t \in \mathbf{F} \mid s \cup t \in X\} \in \mathscr{V}$. As $\mathscr{V}$ is closed under finite intersections, we infer that

$$
\left\{t \in \mathbf{F} \mid \text { for every } s \in \mathrm{FU}\left(\left\{s_{0}, \ldots, s_{m-1}\right\}\right), s \cup t \in X\right\} \in \mathscr{V} .
$$

Let $Q$ consist of those $a \in A$ such that, for some $(f, g) \in \mathscr{H}, \min (\dot{g}(a)) \leqslant$ $\max \left(\dot{g}\left(s_{0} \cup \cdots \cup s_{m-1}\right)\right)$, and observe that $Q$ is finite because $\mathscr{H}$ is. As $\mathscr{V} \in \Psi$, we have FU $(A-Q) \in \mathscr{V}$ and therefore, by the preceding display,

$$
X^{\prime \prime}=\left\{t \in \mathrm{FU}(A-Q) \mid \text { for every } s \in \mathrm{FU}\left(\left\{s_{0}, \ldots, s_{m-1}\right\}\right), s \cup t \in X\right\} \in \mathscr{V} \text {. }
$$

The choice of $Q$ ensures that, for $s$ and $t$ as in the definition of $X^{\prime \prime}$ and for $(f, g) \in \mathscr{H}, \max (\dot{g}(s))<\min (\dot{g}(t))$. Since $\mathscr{V} \in \Psi, X^{\prime \prime}$ is $(\mathscr{H}, m)$-adequate; let $t_{0}, \ldots, t_{m-1}$ witness this, and let $u_{i}=s_{i} \cup t_{i}$ for $i<m$. Then every nonempty union of $u_{i}$ 's has the form $s \cup t$ where $s \in \mathrm{FU}\left(\left\{s_{0}, \ldots, s_{m-1}\right\}\right)$ and $t \in$ $\mathrm{FU}\left(\left\{t_{0}, \ldots, t_{m-1}\right\}\right) \subseteq X^{\prime \prime}$, so $s \cup t \in X$. Furthermore, for each $(f, g) \in \mathscr{H}$, $f\left(\min \left(\dot{g}\left(u_{i}\right)\right)\right)$ and $f\left(\max \left(\dot{g}\left(u_{i}\right)\right)\right)$ are independent of $i$, being equal respectively to $f\left(\min \left(\dot{g}\left(s_{i}\right)\right)\right)$ and $f\left(\max \left(\dot{g}\left(t_{i}\right)\right)\right)$, which are independent of $i$. Thus, $u_{0}, \ldots, u_{m-1}$ witness that $X$ is $(\mathscr{H}, m)$-adequate.

It remains to prove that, for every finite $Q \subseteq A, \operatorname{FU}(A-Q) \in \mathscr{X}$. For any $s \in \mathrm{FU}(A-Q)$, the set $\{t \in \mathbf{F} \mid s \cup t \in \mathrm{FU}(A-Q)\}$ includes $\mathrm{FU}(A-Q)$ and is therefore in $\mathscr{V}$, as $\mathscr{V} \in \Psi$. Thus, $\{s \in \mathbf{F} \mid\{t \in \mathbf{F} \mid s \cup t \in \mathrm{FU}(A-Q)\} \in \mathscr{V}\}$ includes $\mathrm{FU}(A-Q)$ and is therefore in $\mathscr{W}$, as $\mathscr{W} \in \Psi$. This means that $\mathrm{FU}(A-Q)$ $\in \mathscr{X}$, as required.

It is clear from the definition of $\Psi$ that it is a closed and therefore compact subset of the topological space $\beta \mathbf{F}$. This observation, the preceding two lemmas, the one-sided continuity of $\oplus$ and the associativity of $\oplus$ show that $(\Psi, \oplus)$ is a compact left (or right, depending on one's convention) topological semigroup. By a standard theorem $[4,8,10]$ about such semigroups, it contains an idempotent element. Fix some $\mathscr{V} \in \Psi$ with $\mathscr{V} \oplus \mathscr{V}=\mathscr{V}$.

LEMMA 4F. If $\mathrm{FU}(A)$ is partitioned into two pieces then there is a pairwise disjsoint family $B \subseteq \mathrm{FU}(A)$ such that $\mathrm{FU}(B)$ is $\mathscr{C}$-adequate and lies in a single piece of the partition. 
Proof. Since $\mathscr{C}$ is countable, there are only countably many pairs $(\mathscr{H}, m)$ with $\mathscr{H}$ a finite subset of $\mathscr{C}$ and $m \in \omega$. Fix an enumeration of these pairs in an $\omega$-sequence. Let $X$ be the piece of the given partition that is in the idempotent ultrafilter $\mathscr{V}$. ( $X$ exists because $\mathscr{V}$ is an ultrafilter containing $\mathrm{FU}(A)$.)) Call a set $s \in \mathrm{FU}(A)$ good if $s \in X$ and

$$
Y_{s}=\{t \in \mathrm{FU}(A) \mid s \cup t \in X\} \in \mathscr{V} .
$$

We shall inductively construct an infinite sequence $b_{0}, b_{1}, \ldots$ of pairwise disjoint elements of $\mathrm{FU}(A)$ such that every nonempty finite union of them is good and such that if $B$ is the set of all these $b_{i}$ then $\mathrm{FU}(B)$ is $\mathscr{b}$-adequate; this will establish the lemma.

Suppose that, at the $n$th stage of the construction, we have already defined pairwise disjoint $b_{0}, b_{1}, \ldots, b_{k} \in \mathrm{FU}(A)$ such that all nonempty unions of them are good. Then $\mathscr{V}$ contains the sets

$$
\begin{aligned}
& Z_{1}=X \cap \bigcap_{s} Y_{s} \text { where } s \text { ranges over } \mathrm{FU}\left(\left\{b_{0}, \ldots, b_{k}\right\}\right), \\
& Z_{2}=\left\{t \in \mathrm{FU}(A) \mid\left\{u \in \mathrm{FU}(A) \mid t \cup u \in Z_{1}\right\} \in \mathscr{V}\right\}, \text { and } \\
& Z_{3}=\mathrm{FU}(A-Q) \text { where } Q=\left\{a \in A \mid a \subseteq b_{0} \cup \cdots \cup b_{k}\right\} .
\end{aligned}
$$

(For $Z_{2}$, we used that $\mathscr{V}$ is idempotent and contains $Z_{1}$.) Let $(\mathscr{H}, m)$ be the $n$th pair in our fixed enumeration. Since $\mathscr{V} \in \Psi$, we can find $m$ pairwise disjoint sets witnessing the $(\mathscr{H}, m)$-adequacy of $Z_{1} \cap Z_{2} \cap Z_{3}$. Adjoin these $m$ sets to the list of $b$ 's as $b_{k+1}, \ldots, b_{k+m}$. The extended list of $b$ 's is still pairwise disjoint, as the newly adjoined members are in $Z_{3}$. To see that all nonempty unions from the extended list are good, consider any such union, and write it as $s \cup t$, where $s$ is a union of old $b_{i}$ 's $(i \leqslant k)$ and $t$ is a union of newly adjoined $b_{i}$ 's $(i>k)$. We may assume that $t$ is nonempty, for otherwise the induction hypothesis gives the desired conclusion. Since the newly adjoined $b_{i}$ 's witness the ( $\left.\mathscr{H}, m\right)$-adequacy of $Z_{1} \cap Z_{2} \cap Z_{3}$, we have, in particular, $t \in Z_{1} \cap Z_{2}$. From $t \in Z_{1}$, it follows that $s \cup t \in X$. From $t \in Z_{2}$, it follows that $\left\{u \in \mathrm{FU}(A) \mid t \cup u \in Z_{1}\right\} \in \mathscr{V}$, so $\{u \in \mathrm{FU}(A) \mid s \cup t \cup u \in X\} \in$ $\mathscr{V}$. Therefore, $s \cup t$ is good.

After $\omega$ steps, every pair $(\mathscr{H}, m)$ will have been considered, so the list of $b$ 's will include $m$ sets that witness $(\mathscr{H}, m)$-adequacy. Thus, if $B=\left\{b_{i} \mid i \in \omega\right\}$ then $\mathrm{FU}(B)$ is $\mathscr{b}$-adequate. Every $s \in \mathrm{FU}(B)$ is good, by the induction hypothesis carried along during the construction, so $\mathrm{FU}(B) \subseteq X$.

This concludes our work with the fixed countable $\mathscr{C}$ and the fixed $A$ introduced before Lemma $\mathrm{D}$. We are ready to begin the construction of the ultrafilter $\mathscr{U}$ for Theorem 4.

Using the assumption that $\mathrm{CH}$ holds, fix enumerations of length $\boldsymbol{\aleph}_{1}$ of the set of subsets of $\mathbf{F}$, say $\left\{D_{\alpha} \mid \alpha<\aleph_{1}\right\}$, and of the set of one-to-one functions from $\omega$ to $\omega$, say $\left\{g_{\alpha} \mid \alpha<\aleph_{1}\right\}$. We shall define, by induction on $\alpha$, finite-to-one functions $f_{\alpha}$ : $\omega \rightarrow \omega$ and families of pairwise disjoint sets $A_{\alpha} \subseteq \mathbf{F}$ such that:

(1) If $\beta<\alpha$ then there is a finite $Q \subseteq A_{\alpha}$ such that $A_{\alpha}-Q \subseteq \mathrm{FU}\left(A_{\beta}\right)$ (and therefore $\left.\mathrm{FU}\left(A_{\alpha}-Q\right) \subseteq \mathrm{FU}\left(A_{\beta}\right)\right)$. 
(2) $\mathrm{FU}\left(A_{\alpha+1}\right)$ is either included in $D_{\alpha}$ or disjoint from $D_{\alpha}$.

(3) $\mathrm{FU}\left(A_{\alpha}\right)$ is $\mathscr{C}_{\alpha}$-adequate, where $\mathscr{C}_{\alpha}=\left\{\left(f_{\beta}, g_{\beta}\right) \mid \beta<\alpha\right\}$.

Two observations will be useful in the course of this construction. First, if (1) holds for a particular pair $\beta<\alpha$ and also for the pair $\gamma<\beta$, then it holds for the pair $\gamma<\alpha$ also. Indeed, if $A_{\alpha}-Q^{\prime} \subseteq \mathrm{FU}\left(A_{\beta}\right)$ and $A_{\beta}-Q^{\prime \prime} \subseteq \mathrm{FU}\left(A_{\gamma}\right)$, then $A_{\alpha}-Q$ $\subseteq \mathrm{FU}\left(A_{\gamma}\right)$, where $Q$ consists of the members of $Q^{\prime}$ and those other members of $A_{\alpha}$ that have subsets in $Q^{\prime \prime}$; there are only finitely many of the latter because $Q^{\prime \prime}$ is finite and $A_{\alpha}$ is a pairwise disjoint family. Second, in verifying that a set of the form $\mathrm{FU}(A)$ is $\mathscr{C}$-adequate, we can replace the requirement, in the definition of adequacy, that all nonempty unions of the witnesses $s_{i}$ are in $\mathrm{FU}(A)$ by the simpler requirement that the witnesses themselves are in $\mathrm{FU}(A)$, since $\mathrm{FU}(A)$ is closed under finite nonempty unions.

The induction will be arranged so that $f_{\alpha}$ is defined after $A_{\alpha}$ but before $A_{\alpha+1}$. It will be convenient to view the definition of $f_{\alpha}$ and $A_{\alpha+1}$ as constituting together the stage $\alpha+1$ of our construction, so that at limit (and zero) stages we define only $A$ 's not $f$ 's.

At stage 0 , we set $A_{0}=\{\{n\} \mid n \in \omega\}$, so $\mathrm{FU}\left(A_{0}\right)=\mathbf{F}$. Induction hypotheses (1) and (2) are vacuous and (3) is trivial at this stage.

At a successor stage $\alpha=\gamma+1$, assume that we have already defined $A_{\beta}$ for $\beta \leqslant \gamma$ and $f_{\beta}$ for $\beta<\gamma$ in accordance with (1), (2), and (3). In particular, $\mathscr{C}_{\gamma}$ is defined and $\mathrm{FU}\left(A_{\gamma}\right)$ is $\mathscr{C}_{\gamma}$-adequate. We shall first define $f_{\gamma}$ (and thereby define $\left.\mathscr{C}_{\alpha}=\mathscr{C}_{\gamma} \cup\left\{\left(f_{\gamma}, g_{\gamma}\right)\right\}\right)$ so that $\mathrm{FU}\left(A_{\gamma}\right)$ is $\mathscr{C}_{\alpha}$-adequate; thereafter, we shall define $A_{\alpha}$.

Since $\gamma<\boldsymbol{\aleph}_{1}, \mathscr{C}_{\gamma}$ is countable, so there are only countably many pairs $(\mathscr{H}, m)$ where $\mathscr{H}$ is a finite subset of $\mathscr{C}_{\gamma}$ and $m \in \omega$. List these pairs in an $\omega$-sequence. We shall associate to each such pair $(\mathscr{H}, m)$ certain sets $s_{0}, \ldots, s_{m-1}$ witnessing the $(\mathscr{H}, m)$-adequacy of $\mathrm{FU}\left(A_{\gamma}\right)$, in such a way that the sets associated to different pairs are disjoint. (Sets associated to the same pair are disjoint by definition of adequacy.) We do this by induction along our list of pairs. When pair $(\mathscr{H}, m)$ is to be treated, let $N$ be the number of $a \in A_{\gamma}$ such that $a$ is included in one of the (finitely many) sets already associated to earlier pairs in the list. $\operatorname{As} \operatorname{FU}\left(A_{\gamma}\right)$ is $\mathscr{C}_{\gamma}$-adequate, choose sets that witness its $(\mathscr{H}, m+N)$-adequacy. At most $N$ of these $m+N$ sets intersect any of the sets already associated to earlier pairs, so at least $m$ do not. Choose any $m$ of these and associate them to $(\mathscr{H}, m)$.

Define $f_{\gamma}$ so that, if $s$ is associated to the $k$ th pair $(\mathscr{H}, m)$ in our list, then $f_{\gamma}$ takes the value $k$ at all points in $g_{\gamma}[s]$. This is possible since all the $s$ 's are disjoint and $g_{\gamma}$ is one-to-one. Extend $f_{\gamma}$ to all of $\omega$ by setting $f_{\gamma}(x)=x$ if $x$ belongs to no $g_{\gamma}[s]$ with $s$ as above. Then $f_{\gamma}$ is a finite-to-one function on $\omega$, and the sets associated to any $(\mathscr{H}, m)$ witness not only the $(\mathscr{H}, m)$-adequacy but the $(\mathscr{H}$ $\left.\cup\left\{\left(f_{\gamma}, g_{\gamma}\right)\right\}, m\right)$-adequacy of $\mathrm{FU}\left(A_{\gamma}\right)$. Thus, $\mathrm{FU}\left(A_{\gamma}\right)$ is $\mathscr{C}_{\alpha}$-adequate.

Apply Lemma $4 \mathrm{~F}$ with $A_{\gamma}$ as $A, \mathscr{C}_{\alpha}$ as $\mathscr{C}$, and $\left\{D_{\gamma}, \mathbf{F}-D_{\gamma}\right\}$ as the partition. (The hypotheses on $\mathscr{C}$ and $A$ before Lemma 4D are satisfied.) The lemma produces a pairwise disjoint family $B \subseteq \mathrm{FU}\left(A_{\gamma}\right)$ such that $\mathrm{FU}(B)$ is $\mathscr{C}_{\alpha}$-adequate and either included in $D_{\gamma}$ or disjoint from $D_{\gamma}$. Set $A_{\alpha}=B$. Induction hypotheses (2) and (3) are clearly preserved, and (1) is trivial (with $Q$ empty) for $\beta=\gamma$. The first 
preliminary observation (right after (3)) and the induction hypothesis (1) with $\gamma$ in place of $\alpha$ then yield (1) for all $\beta<\alpha$.

Finally, suppose that $\alpha$ is a limit ordinal and that $A_{\beta}$ and $f_{\beta}$ have been defined for all $\beta<\alpha$ in accordance with (1)-(3). We must define $A_{\alpha}$ so that these induction hypotheses are preserved. We can ignore (2), since it refers only to $A$ 's with successor subscripts.

Lemma 4G. For each $\beta<\alpha, \mathrm{FU}\left(A_{\beta}\right)$ is $\mathscr{C}_{\alpha}$-adequate.

Proof. Let $\mathscr{H}$ be a finite subset of $\mathscr{C}_{\alpha}$ and let $m \in \omega$. Pick a $\gamma$ such that $\beta<\gamma<\alpha$ and $\mathscr{H} \subseteq \mathscr{C}_{\gamma}$. By induction hypothesis (1), pick a finite $Q \subseteq A_{\gamma}$ such that $\mathrm{FU}\left(A_{\gamma}-Q\right) \subseteq \mathrm{FU}\left(A_{\beta}\right)$, and let $q$ be the cardinality of $Q$. By induction hypothesis (3), find $m+q$ sets to witness the ( $\mathscr{H}, m+q)$-adequacy of $\mathrm{FU}\left(A_{\gamma}\right)$. Then some $m$ of these sets have no subsets in $Q$ and are therefore in $\operatorname{FU}\left(A_{\beta}\right)$. They witness the $(\mathscr{H}, m)$-adequacy of $\mathrm{FU}\left(A_{\beta}\right)$.

Enumerate in an $\omega$-sequence all the pairs $(\mathscr{H}, m)$ with $\mathscr{H}$ a finite subset of $\mathscr{C}_{\alpha}$ and $m \in \omega$; also enumerate a strictly increasing $\omega$-sequence of ordinals $\beta(n)$ with limit $\alpha$. We intend to associate to the $k$ th pair $(\mathscr{H}, m) m$ sets that witness the ( $\mathscr{H}, m)$-adequacy of $\bigcap_{n \leqslant k} \mathrm{FU}\left(A_{\beta(n)}\right)$, and we intend to do this so that all the associated sets are disjoint. To do this, notice first that, by applying (1) with $\beta(k)$ as $\alpha$ and various $\beta(n), n<k$, as $\beta$, we obtain a finite $Q$ such that $\bigcap_{n \leqslant k} \operatorname{FU}\left(A_{\beta(n)}\right)$ includes $\mathrm{FU}\left(A_{\beta(k)}-Q\right)$ and is therefore $\mathscr{C}_{\alpha}$-adequate because $\mathrm{FU}\left(A_{\beta(k)}\right)$ is by Lemma $4 \mathrm{G}$. The $\mathscr{C}_{\alpha}$-adequacy of $\bigcap_{n \leqslant k} \mathrm{FU}\left(A_{\beta(n)}\right)$ allows us to produce the required associated sets just as in the second paragraph of the successor case. Let $A_{\alpha}$ consist of all the sets associated to the various $(\mathscr{H}, m)$. Those associated to a particular $(\mathscr{H}, m)$ witness that $\mathrm{FU}\left(A_{\alpha}\right)$ is $(\mathscr{H}, m)$-adequate, so $\mathrm{FU}\left(A_{\alpha}\right)$ is $\mathscr{C}_{\alpha}$-adequate as required by (3). Finally, we check (1). The construction ensures that all but finitely many of the sets in $A_{\alpha}$ (namely all but those associated to the first $n$ pairs) are in FU $\left(A_{\beta(n)}\right)$, so (1) holds when $\beta=\beta(n)$ for some $n$. Since the $\beta(n)$ are cofinal in $\alpha$, (1) holds for all other $\beta<\alpha$, by the induction hypothesis (with a suitable $\beta(n)$ in place of $\alpha$ ) and the first observation after (3).

This completes the construction of the sets $A_{\alpha}$ and the functions $f_{\alpha}$. It follows from (1) that the family $\left\{\mathrm{FU}\left(A_{\alpha}\right) \mid \alpha<\aleph_{1}\right\}$ has the finite intersection property, and then it follows from (2) that this family is a basis for an ultrafilter $\mathscr{U}$. Since the sets in this basis all have the form $\mathrm{FU}(A), \mathscr{U}$ is a union ultrafilter. It remains to prove that, if $g: \omega \rightarrow \omega$ is one-to-one then neither $\max (\dot{g}(\mathscr{U}))$ nor $\min (\dot{g}(\mathscr{U}))$ is a $Q$-point.

Exactly as in the proof of Lemma $4 \mathrm{G}$ (with $\alpha$ changed to $\boldsymbol{\aleph}_{1}$ ), we see that all of the sets $\mathrm{FU}\left(A_{\alpha}\right)$ in our basis for $\mathscr{U}$, and therefore all sets in $\mathscr{U}$ are $\mathscr{C}$-adequate, where $\mathscr{C}=\left\{\left(f_{\beta}, g_{\beta}\right) \mid \beta<\aleph_{1}\right\}$. An arbitrary one-to-one $g: \omega \rightarrow \omega$ is $g_{\beta}$ for some $\beta$. Write $f$ for the finite-to-one function $f_{\beta}$, and observe that $\mathscr{C}$-adequacy requires each set $X \in \mathscr{U}$ to be $(\{(f, g)\}, 2)$-adequate. In particular, $X$ contains two disjoint sets $s$ and $t$ such that $f(\min (\dot{g}(s)))=f(\min (\dot{g}(t)))$ and similarly for max. Thus, each set in $\min (\dot{g}(\mathscr{U}))$ contains two distinct elements with the same $f$-image, so $\min (\dot{g}(\mathscr{U}))$ is not a $Q$-point, and, similarly, neither is $\max (\dot{g}(\mathscr{U}))$. 
We show next how to modify the preceding proof of Theorems $4,4^{\prime}$, and $4^{\prime \prime}$ so as to use only MA rather than $\mathrm{CH}$. The crucial difference, of course, is that the enumeration of the subsets $D_{\alpha}$ of $\mathbf{F}$ and of the one-to-one functions $g_{\alpha}: \omega \rightarrow \omega$ are of length $c=2^{\aleph_{0}}$, which is no longer known to be $\aleph_{1}$. Thus, the inductive construction of the ultrafilter $\mathscr{U}$ now has length $c$. Thus, the sets $\mathscr{C}_{\gamma}=\left\{\left(f_{\beta}, g_{\beta}\right) \mid \beta\right.$ $<\gamma\}$ occurring in the construction are not necessarily countable but merely of cardinality $<c$. It is therefore necessary, in Lemmas $4 \mathrm{D}$ to $4 \mathrm{~F}$, to allow $\mathscr{C}$ to have any cardinality $<c$, since Lemma $4 \mathrm{~F}$ is applied with $\mathscr{C}_{\gamma}$ in the role of $\mathscr{C}$. It is also necessary to modify the construction of $f_{\gamma}$ at successor stages $\gamma+1$ and the construction of $A_{\alpha}$ at limit stages $\alpha$, since these depend on enumerating $\mathscr{C}_{\gamma}$ or $\mathscr{C}_{\alpha}$ in an $\omega$-sequence and the latter also uses an $\omega$-sequence of ordinals with limit $\alpha$. All three of these modifications involve applications of MA, and we now consider each in turn.

In Lemmas $4 \mathrm{D}$ through $4 \mathrm{~F}$, let us weaken the assumption that $\mathscr{C}$ is countable to require only that $\mathscr{C}$ have cardinality $<c$. The proofs of Lemmas $4 \mathrm{D}$ and $4 \mathrm{E}$ did not use the countability hypothesis, so these proofs remain correct, and we have, as before, an ultrafilter $\mathscr{V} \in \Psi$ with $\mathscr{V} \oplus \mathscr{V}=\mathscr{V}$. The proof of Lemma $4 \mathrm{~F}$ is no longer valid, because it involved listing all the pairs $(\mathscr{H}, m)$, with $\mathscr{H}$ a finite subset of $\mathscr{C}$ and $m \in \omega$, in an $\omega$-sequence. The number of these pairs is no longer known to be countable, though it is $<c$ (because the cardinality of $\mathscr{C}$ is). We replace the proof of Lemma $4 \mathrm{~F}$ with the following MA argument.

Let $P$ be the notion of forcing whose elements are finite pairwise disjoint subsets $p=\left\{b_{0}, \ldots, b_{k}\right\} \subseteq \mathrm{FU}(A)$ such that every nonempty union of members of $p$ is good; here "good" is defined exactly as in the old proof of Lemma 4F. $P$ is ordered by reverse inclusion; i.e., extensions are supersets. The second paragraph of the old proof shows how to extend any condition to one for which $\mathrm{FU}(p)$ is $(\mathscr{H}, m)$ adequate for a prescribed pair $(\mathscr{H}, m)$. Thus, the sets

$$
\Delta(\mathscr{H}, m)=\{p \in P \mid \mathrm{FU}(p) \text { is }(\mathscr{H}, m) \text {-adequate }\}
$$

are dense in $P$. Since there are fewer than $c$ pairs $(\mathscr{H}, m)$, and since $P$ trivially satisfies the countable antichain condition (being countable), MA provides a filter $G \subseteq P$ meeting every $\Delta(\mathscr{H}, m)$. Then the union of all the conditions $p \in G$ is a pairwise disjoint family $B \subseteq \mathrm{FU}(A)$ such that $\mathrm{FU}(B)$ consists of good sets (because $G$ is a filter) and therefore lies in the single piece $X$ of the given partition, and such that $\mathrm{FU}(B)$ is $\mathscr{C}$-adequate (as $G$ meets every $\Delta(\mathscr{H}, m)$ ). This completes the new proof of Lemma $4 \mathrm{~F}$.

We turn next to the construction of $f_{\gamma}$ at a successor stage $\gamma+1$ in our inductive construction of $\mathscr{U}$. We have that $\mathrm{FU}\left(A_{\gamma}\right)$ is $\mathscr{C}_{\gamma}$-adequate and we want a finite-to-one function $f_{\gamma}$ such that $\mathrm{FU}\left(A_{\gamma}\right)$ is also $\mathscr{C}_{\gamma+1}$-adequate, where $\mathscr{C}_{\gamma+1}=\mathscr{C}_{\gamma} \cup\left\{\left(f_{\gamma}, g_{\gamma}\right)\right\}$. Let $P$ be the notion of forcing whose elements are pairs $\langle p, n\rangle$ such that $p$ is a function from a finite subset of $\omega$ into $\omega$ and $n \in \omega$. (We think of $\langle p, n\rangle$ as giving the following information about the desired $f_{\gamma}$ : It extends $p$, and $f_{\gamma}(x) \geqslant n$ for all $x \notin$ domain $(p)$. The latter is to ensure that $f_{\gamma}$ will be finite-to-one.) The ordering of $P$ is defined by calling $\left\langle p^{\prime}, n^{\prime}\right\rangle$ an extension of $\langle p, n\rangle$ if $p^{\prime}$ extends $p$ (as 
functions, i.e., $\left.p \subseteq p^{\prime}\right), n^{\prime} \geqslant n$, and $p^{\prime}(x) \geqslant n$ for all $x \in \operatorname{domain}\left(p^{\prime}\right)-\operatorname{domain}(p)$. $P$ is countable, so the countable antichain condition is trivially satisfied. We consider three sorts of dense subsets of $P$.

$$
\begin{aligned}
& \Delta_{1}(k)=\{\langle p, n\rangle \in P \mid k \in \text { domain }(p)\} \quad \text { for } k \in \omega . \\
& \Delta_{2}(k)=\{\langle p, n\rangle \in P \mid n>k\} \text { for } k \in \omega . \\
& \Delta_{3}(\mathscr{H}, m)=\left\{\langle p, n\rangle \in P \mid \text { there exist } s_{0}, \ldots, s_{m-1}\right. \text { witnessing the } \\
&(\mathscr{H}, m) \text {-adequacy of } \mathrm{FU}\left(A_{\gamma}\right) \text { such that } p \text { is } \\
&\text { defined and constant on } \left.g_{\gamma}\left[s_{0} \cup \cdots \cup s_{m-1}\right]\right\} \\
& \text { for } \mathscr{H} \text { a finite subset of } \mathscr{C}_{\gamma} \text { and } m \in \omega .
\end{aligned}
$$

The density of the sets $\Delta_{1}(k)$ and $\Delta_{2}(k)$ is obvious. For $\Delta_{3}(\mathscr{H}, m)$, proceed as follows. Given $\langle p, n\rangle$, let $N$ be the cardinality of the domain of $p$. As $\operatorname{FU}\left(A_{\gamma}\right)$ is $\mathscr{C}_{\gamma}$-adequate, find $m+N$ sets witnessing the $(\mathscr{H}, m+N)$-adequacy of $\mathrm{FU}\left(A_{\gamma}\right)$. As these sets are disjoint, at most $N$ of them intersect $g_{\gamma}^{-1}$ [domain $(p)$ ], leaving at least $m$ that do not. Take $m$ that do not, and call them $s_{0}, \ldots, s_{m-1}$. Then $p$ is not defined at any point of $g\left[s_{0} \cup \cdots \cup s_{m-1}\right]$, so we can extend it to take the value $n$ at all these points. Thus we obtain an extension $\left\langle p^{\prime}, n\right\rangle \in \Delta_{3}(\mathscr{H}, m)$ of $\langle p, n\rangle$.

MA provides a filter $G$ in $P$ meeting all the dense sets $\Delta_{1}(k), \Delta_{2}(k)$, and $\Delta_{3}(\mathscr{H}, m)$, as there are fewer than $c$ of these. The union of the first components $p$ of all the pairs $\langle p, n\rangle \in G$ is a function $f_{\gamma}: \omega \rightarrow \omega$ because $G$ is a filter and meets each $\Delta_{1}(k)$. Furthermore, for each $k, G$ meets $\Delta_{2}(k)$, i.e., $G$ contains some $\left\langle p_{0}, n_{0}\right\rangle$ with $n_{0}>k$. Then, for every $\langle p, n\rangle \in G$, there is a common extension $\left\langle p^{\prime}, n^{\prime}\right\rangle$ of $\left\langle p_{0}, n_{0}\right\rangle$ and $\langle p, n\rangle$. So if $x \in \operatorname{domain}(p)-\operatorname{domain}\left(p_{0}\right)$, then $p(x)=p^{\prime}(x) \geqslant n_{0}$ $>k$. This shows that $f_{\gamma}$ never takes the value $k$ at points outside domain $\left(p_{0}\right)$. As $k$ was arbitrary, ${ }_{{ }^{\prime}}$ is finite-to-one. Finally, if $\langle p, n\rangle \in G \cap \Delta_{3}(\mathscr{H}, m)$ then the sets $s_{0}, \ldots, s_{m-1}$ required in the definition of " $\langle p, n\rangle \in \Delta_{3}(\mathscr{H}, m)$ " witness the $(\mathscr{H}, m)$-adequacy of $\mathrm{FU}\left(A_{\gamma}\right)$ and, in addition, have $p$ and therefore $f_{\gamma}$ constant on $g_{\gamma}\left[s_{0} \cup \cdots \cup s_{m-1}\right]$. They therefore witness the $\left(\mathscr{H} \cup\left\{\left(f_{\gamma}, g_{\gamma}\right)\right\}, m\right)$-adequacy of $\mathrm{FU}\left(A_{\gamma}\right)$. Thus, $f_{\gamma}$ has all the required properties.

Finally, we turn to the construction of $A_{\alpha}$ at limit stages $\alpha$. The proof of Lemma $4 \mathrm{G}$ needs no changes, so $\mathrm{FU}\left(A_{\beta}\right)$ is $\mathscr{C}_{\alpha}$-adequate for all $\beta<\alpha$. We must find a disjoint family $A_{\alpha} \subseteq \mathbf{F}$ such that $\mathrm{FU}\left(A_{\alpha}\right)$ is $\mathscr{C}_{\alpha}$-adequate and, for each $\beta<\alpha$, there is a finite set $Q$ with $A_{\alpha}-Q \subseteq \mathrm{FU}\left(A_{\beta}\right)$. Define a notion of forcing $P$ to consist of pairs $\langle p, s\rangle$ where $p$ is a finite pairwise disjoint subfamily of $\mathbf{F}$ and $s$ is a finite subset of the ordinal $\alpha$. (We think of $\langle p, s\rangle$ as giving the following information about $A_{\alpha}: p \subseteq A_{\alpha}$, and $A_{\alpha}-p \subseteq \mathrm{FU}\left(A_{\beta}\right)$ for every $\beta \in s$.) An extension of $\langle p, s\rangle$ is $\left\langle p^{\prime}, s^{\prime}\right\rangle$ such that $p^{\prime} \supseteq p, s^{\prime} \supseteq s$, and $p^{\prime}-p \subseteq \mathrm{FU}\left(A_{\beta}\right)$ for all $\beta \in s$. There are only countably many first components $p$ for conditions $\langle p, s\rangle$, and any two conditions with the same first component are compatible (by taking the union of the second components), so $P$ satisfies the countable antichain condition. Consider the sets

$$
\Delta_{1}(\beta)=\{\langle p, s\rangle \in P \mid \beta \in s\} \quad \text { for } \beta<\alpha,
$$


and

$$
\begin{aligned}
& \Delta_{2}(\mathscr{H}, m)=\{\langle p, s\rangle \in P \mid \mathrm{FU}(p) \text { is }(\mathscr{H}, m) \text {-adequate }\} \\
& \text { for } \mathscr{H} \text { a finite subset of } \mathscr{C}_{\alpha} \text { and } m \in \omega .
\end{aligned}
$$

Obviously, each $\Delta_{1}(\beta)$ is dense in $P$. To see that $\Delta_{2}(\mathscr{H}, m)$ is also dense, let $(\mathscr{H}, m)$ and $\langle p, s\rangle$ be given. Let $\gamma<\alpha$ be an upper bound for the finite set $s$. By induction hypothesis (1) of our construction, we have, for each $\beta \in s$, a finite set $Q_{\beta} \subseteq A_{\gamma}$ with $A_{\gamma}-Q_{\beta} \subseteq \mathrm{FU}\left(A_{\beta}\right)$. Let $Q$ be the union of these (finitely many) $Q_{\beta}$ 's. Then $\mathrm{FU}\left(A_{\gamma}-Q\right) \subseteq \mathrm{FU}\left(A_{\beta}\right)$ for all $\beta \in s$, and $\mathrm{FU}\left(A_{\gamma}-Q\right)$ is $\mathscr{C}_{\alpha}$-adequate because $\mathrm{FU}\left(A_{\gamma}\right)$ is. Let $s_{0}, \ldots, s_{m-1}$ witness the $(\mathscr{H}, m)$-adequacy of FU $\left(A_{\gamma}-Q\right)$. Then $\left\langle p \cup\left\{s_{0}, \ldots, s_{m-1}\right\}, s\right\rangle$ is an extension of $\langle p, s\rangle$ and is in $\Delta_{2}(\mathscr{H}, m)$.

MA provides a filter $G$ in $P$ meeting all the dense sets $\Delta_{1}(\beta)$ and $\Delta_{2}(\mathscr{H}, m)$, because there are fewer than $c$ of them. The union of the first components $p$ of all the conditions $\langle p, s\rangle \in G$ is a disjooint subfamily $A_{\alpha}$ of F. It is $\mathscr{C}_{\alpha}$-adequate because $G$ meets every $\Delta_{2}(\mathscr{H}, m)$. Furthermore, for each $\beta<\alpha$, there is $\left\langle p_{0}, s_{0}\right\rangle \in G \cap$ $\Delta_{1}(\beta)$, so $\beta \in s_{0}$. For each $\langle p, s\rangle \in P$, there is a common extension $\left\langle p^{\prime}, s^{\prime}\right\rangle$ of $\left\langle p_{0}, s_{0}\right\rangle$ and $\langle p, s\rangle$. This implies that $p-p_{0} \subseteq p^{\prime}-p_{0} \subseteq \mathrm{FU}\left(A_{\beta}\right)$. Therefore $A_{\alpha}-$ $p_{0} \subseteq \mathrm{FU}\left(A_{\beta}\right)$. Thus, $A_{\alpha}$ has all the required properties.

This completes the modification of the proofs to use MA instead of $\mathrm{CH}$. Observe that, in two of the three applications of MA, the notion of forcing $P$ was countable. In the third, it was uncountable but $\sigma$-centered, i.e., the union of countably many subsets such that every finitely many elements in any single subset have a common extension; the subsets are those in which $p$ is constant while $s$ varies. Thus, the entire proof needs MA only for $\sigma$-centered notions of forcing. It is a theorem of Bell [2] that this special case of MA is equivalent to the assertion, known as $P(c)$, that, if $\mathscr{F}$ is a family of fewer than $c$ subsets of $\omega$ and if every finite subfamily has infinite intersection, then there is an infinite $A \subseteq \omega$ such that $A-B$ is finite for all $B \in \mathscr{F}$. Summarizing, we have the following result

THEOREM 5. The conclusions of Theorems 4, 4', and 4" hold when the hypothesis $C H$ is weakened to $M A$ or even to $P(c)$.

5. Miscellany. Theorem 2 allows us to simplify Theorem 4.2 of [3] by deleting in part (f) the clause that $\min (\mathscr{U})$ is a $P$-point, since this is always true. Thus, an ordered-union untrafilter is stable if and only if the substructure generated by [min] in the ultrapower $\mathscr{U}$-prod $\omega$ is an initial segment of this ultrapower.

The definition of stability for ordered-union ultrafilters in [3] can be applied as well to union ultrafilters. A union ultrafilter $\mathscr{U}$ is stable if, whenever it contains $\mathrm{FU}\left(A_{n}\right)$ for each of countably many families $A_{n}$ of pairwise disjoint sets in $\mathbf{F}$, then it also contains $\mathrm{FU}(A)$ for another such family $A$ such that, for each $n, A$ has a finite subset $Q$ with $A-Q \subseteq \mathrm{FU}\left(A_{n}\right)$. The union ultrafilters constructed in $\S 4$ are clearly stable, by clause (1) of the induction hypothesis. Thus, stability does not imply additive isomorphism to an ordered-union ultrafilter. We have not investigated whether stability in this more general setting implies any other interesting properties (as in $\S 4$ of [3] for the ordered-union setting). 
The proof of the "min" case of Theorem 2 can be modified to give the following partition relation (which in turn implies that $\min (\mathscr{U})$ is a $P$-point, as one sees by partitioning the pairs $\{a, b\}$ with $a<b$ according to whether $a<f(b)$ ).

THEOREM 6. Let $\mathscr{U}$ be a union ultrafilter, and let the set $[\omega]^{2}$ of two-element subsets of $\omega$ be partitioned into two pieces. Then there exists sets $A \in \max (\mathscr{U})$ and $B \in$ $\min (\mathscr{U})$ such that one of the partition pieces contains all the pairs $\{a, b\}$ with $a \in A$, $b \in B$, and $a<b$.

Proof. Let the two pieces of the partition of $[\omega]^{2}$ be $C_{0}$ and $C_{1}$. For each $s \in \mathbf{F}$, let $h(s)$ be the number of consecutive pairs $(x, y)$ of $s$ such that $\{x, y\} \in C_{1}$. Because $\mathscr{U}$ is a union ultrafilter, there is an infinite pairwise disjoint family $S \subseteq \mathbf{F}$ such that $\mathrm{FU}(S) \in \mathscr{U}$ and $h(s)$ has the same parity for all $s \in \mathrm{FU}(S)$.

Suppose $s, t \in S$ and $\max (s)<\min (t)$. Then

$$
h(s \cup t)=h(s)+h(t)+ \begin{cases}1 & \text { if }\{\max (s), \min (t)\} \in C_{1}, \\ 0 & \text { if }\{\max (s), \min (t)\} \in C_{0} .\end{cases}
$$

Case 1. $h(s)$ is odd for all $s \in \mathrm{FU}(S)$.

Then the last term in $(*)$ must be 1 . So, if $a \in \max [\mathrm{FU}(S)], b \in \min [\mathrm{FU}(S)]$, and $a<b$, then $\{a, b\} \in C_{1}$.

Case 2. $h(s)$ is even for all $s \in \mathrm{FU}(S)$.

Then the last term in (*) must be 0 . So if $a \in \max [\mathrm{FU}(S)], b \in \min [\mathrm{FU}(S)]$, and $a<b$, then $\{a, b\} \in C_{0}$.

Thus, in either case, $A=\max [\mathrm{FU}(S)]$ and $B=\min [\mathrm{FU}(S)]$ are as required.

Finally, we must point out that the construction in $\$ 4$ leaves open the question whether every union ultrafilter is isomorphic to an ordered-union one. In other words, can we delete "additively" from the statement of Theorem 4 ?

\section{REFERENCES}

1. J. Baumgartner, A short proof of Hindman's theorem, J. Combin. Theory (A) 17 (1974), 384-386.

2. M. Bell, On the combinatorial principle P(c), Fund. Math. 114 (1981), 149-157.

3. A. Blass, Ultrafilters related to Hindman's finite-unions theorem and its extensions, Logic and Combinatorics (S. Simpson, ed.), Contemp. Math., vol. 65, Amer. Math. Soc., Providence, R.I., 1987, pp. $89-124$.

4. W. W. Comfort, Ultrafilters-some old and some new results, Bull. Amer. Math. Soc. 83 (1977). 417-455.

5. R. Graham and B. Rothschild, Ramsey's theorem for n-parameter sets, Trans. Amer. Math. Soc. 159 (1971), 257-292.

6. N. Hindman, The existence of certain ultrafilters on $N$ and a conjecture of Graham and Rothschild, Proc. Amer. Math. Soc. 36 (1972), 341-346.

7. Finite sums from sequences within cells of a partition of $N$, J. Combin. Theory (A) 17 (1974), $1-11$.

8. Ultrafilters and combinatorial number theory, Number Theory, Carbondale 1979, (M. Nathanson, ed.), Lecture Notes in Math., vol. 751, Springer-Verlag, Berlin and New York, 1979, pp. $119-184$.

9. Summable ultrafilters and finite sums, Logic and Combinatorics (S. Simpson, ed.), Contemp. Math., vol. 65, Amer. Math. Soc., Providence, R.I., 1987, pp. 263-274. 
10. K. Numakura, On bicompact semigroups, Math. J. Okayama Univ. 1 (1952), 99-108.

11. S. Shelah, Proper forcing, Lecture Notes in Math., vol. 940, Springer-Verlag, Berlin and New York, 1982.

12. E. Wimmers, The Shelah P-point independence theorem, Israel J. Math. 43 (1982) 28-48.

Department of Mathematics, Pennsylvania State University, University Park, Pennsylvania 16802

Department of Mathematics, Howard University, Washington, D. C. 20059 (Current address of Neil Hindman)

Current address (Andreas Blass): Department of Mathematics, University of Michigan, Ann Arbor, Michigan 48109 